\title{
Zur Arbeit E. v. Hippels ïber: „Weitere Erfahrungen über die Ergebnisse der druckentlastenden Operationen bei der Staunnspapille“.
}

\author{
Ton \\ Augenarzt und Dozent Dr. Hermann Davids. \\ uinster in $W$.
}

In seiner Arbeit über: „Weitere Erfahrungen über die Ergebnisse der druckentlastenden Operationen bei der Stauungspapille" erwähnt E. v. Hip pel ${ }^{1}$ ) unter den 3 Fällen, in denen der Balkenstich und die Trepanation gemacht wurden, auch den Fall Feldhaus. Die Mitteilung v. Hippels lautet:

„Feldha us. R. S. $={ }^{5} / 5$, L. S. $={ }^{5} / 15$. 1. VIII. 1913 Balkenstich. Kein Erfolg. 15. IX. 1914 Trepanation. Rückgang der Stauungspapille rechts von 3 auf $1 \mathrm{D}$, links deutliche Abblassung, rechts Spur davon. Die Beobachtung konnte nicht weiter geführt werden, da Patient nach Hause reiste. Ex ist dann noch mehrere Jahre als Rechtsanwalt tätig gewesen. 4 .Jahre später erhielt ich die briefliche Mitteilung, daß der Visus in letzter Zeit schlechter geworden sei. Ob Patient noch lebt, kann ich nicht angeben. In diesem Falle war also die Trepanation dem Balkenstich überlegen und hat einen zweifellosen Erfolg gehabt.

Dieser Fall Feldhaus steht seit 1914 auch in meiner Behandlung bzw. Beobachtung und hat einen so bemerkenswerten weiteren Verlauf genommen, daß die Veröffentlichung meiner Beobachtungen berechtigt erseheinen dürfte, zumal sie eine nicht unwichtige Ergänzung der Mitteilungen v. Hip pels bilden. Nach meinen Aufzeichnungen nahm die Krankheit folgenden Verlauf:

25. V. 1914. Angeblich 1. VIIr. 1913 Balkenstich in Halle wegen Kopfsehmerzen, Druckgefühl im Kopf, Schwindel und wegen drohender Erblindung des linken Auges.

R. Papillengrenze oben und unten verschwommen, Venen geschlängelt und etwas erweitert. Temporale Farbenhemianopsie. $\mathrm{S} .=1,0 . \mathrm{L}$. neuritische Atrophie. S. = Handbewegungen vorm Auge.

15. IX. 1914. Trepanation in Halle aul Veranlassung von A nton und E. v. Hippel. schein.

5. II. 1915. R. S. $=<0,8$. Papille ein wenig geschwollen. L. geringer Licht-

18. XI. 1916. Beiderseits Atrophie nach Stauungspapille. R. S. = Finger in $2^{1 / 2} \mathrm{~m}$. Gläser bessern nicht. L. Amaurose. Trepanationsstelle tritt stark hervor.

Während in der Folgezeit das Sehvermögen des rechten Auges nur langsam weiter verfiel, wurde das Allgemeinbefinden sichtbar immer schlechter. Der Pro- 
laps trat immer stärkex hervor. Beiderseits bestand lejchte Protrusio bulbi. Das Gesicht war leicht cyanotisch. Der Körper war stark abgemagert (vgl. unten), die Körperhaltung war schlaff, gebeugt. Der Gang war langsam, schlürfend, mit gebeugten Knien. Patient konnte nur noch kurze Ausgänge machen; war zeitweilig bettlägerig, hatte rheumatische Schmerzen in den Beinen, Zittern der Hände, war leicht erregt, dann wieder deprimiert, litt an Ohnmachtsanwandlangen und an epileptiformen Anfällen derart, daß mit seinem Ableben gerechnet wurde.

Diese Verschlechterung des Allgemeinzustandes und des Augenbefundes hielt an bis Mitte 1919. Zu dieser Zeit trat ein starker Abflu $B$ von Flüssigkeit aus der Nase ein und zugleich eine erhebliche fortschreitende allgemeine Besserung. Die Flüssigkeit wurde untersucht und erwies sich nach ilirem chemischen Verhalten als Liquor cerebrospinalis.

16. IX. 1919. R. S. $=<0,3 !$ mit $+1,0$ D. sph. $=+0,5$ D cyl. $\uparrow$. L. A maurose.

Allgemeinzustand hat sich erheblich gebessert. Dauernder LiquorabfluB aus der Nase, entweder tropfenweise oder unter Druck in kurzem Strom.

17. IV. 1920. R. A. nicht wesentlich verändert. L. Arterien sehr dünn. Unbestimmter Lichtschein, ganz schwache Pupillenreaktion auf Licht. Patient macht einen frischeren, gesunderen Eindruck, ist bedeutend ruhiger. Haltung und Gang sind straffer.

20. X. 1920. Seit Mai-Juni weitere erhebliche Besserung. Patient fühlt sich wohler, hat keine Anfälle mehr, Stimmung gleichmäßiger, Haltung und Gang normal, geht $30-40 \mathrm{~km}$ ohne Beschwerden. Hirnprolaps bedeutend abgeflacht. Der Liquorabfluß hat seit Anfang 1920 wesentlich nachgelassen.

Beiderseits Spiegelbild unverändert: Neuritische Atrophie, Papillen granweiB l. > r. Grenzen verschwommen, Arterien 1. ganz dünn.

R. S. $=0,7-1,0$ ! Auge ermüdet noch leicht. L. unbestimmter Lichtsehein und unsichere Reaktion der Pupille auf Licht. Außengrenze des rechten Gesichtsfeldes normal, temporale Farbenhemianopsie.

29. XII. 1920. R. S. $=0,9-1,0$ mit $+0,75$ Dcyl $\uparrow$. Nieden Nr. 1. Auge ermüdet noch leicht. Keine Protrusio bulbi mehr. Prolaps flacher. Patient ist als Rechtsanwalt tätig und fühlt sich gesund.

25. X. 1921. Augenbefund unverändert, ebenso das Allgemeinbefinden.

I0. I. 22. Der Liquorabfluß hat inzwischen gänzlich aufgehört. Sonst Zustand völlig unverändert.

Bemerkenswert ist noeh das Körpergewicht des Patienten, das sich dem jeweiligen Zustande anpaBte: Es betrug $1913=198$ Pfund, 1916=106 Pfund $1920=142$ Pfund. -

In zweierlei Hinsicht ist der beschriebene Fall nach meinem Dafürhalten bemerkenswert. Zunächst gehört er zu den Fällen, in denen durch Abfluß von Liquor Druckentlastung und somit eine Art von Selbstheilung eintrat. v. Hip pel²) hat diese seltenen Fälle im Handbuch der gesamten Augenheilkunde zusammengestellt. Es sind die 18 Fälle, die Wolf ${ }^{3}$ ) in seiner Dissertation auf Veranlassung Körners veröffentlichte, und 6 weitere Fälle, die Wolf übersah, nämlich die von Elliotson ${ }^{4}$ ), Paget ${ }^{5}$, Babinski ${ }^{6}$ ), Nothnageli), Emrys Jones ${ }^{8}$ ) und Lucae $e^{9}$ beschriebenen, sodann 1 Fall von Starck ${ }^{10}$ ) und 2 von Coppezix).

Thnen fügt v. Hippel folgende eigene Beobachtung hinzu: 
„Heilung der Stauungspapille duroh Palliativtrepanation für 2 Jahre. Großer Prolaps. Dann Verschlechterung des Visus. Jetzt trat AusfluB aus dex Nase auf. mit Besserung des Sehvermögens für ein Jahr. Nun Verschlimmerung, Doppeltsehen. Ein Jahr später Exitus."

Der Fall Lucae scheint mir strenggenommen nicht hierher zu gehören, da es sich bei ihm nach der mir vorliegenden Arbeit nur um einen 5 Wochen anhaltenden profusen Ausfluß von Liquor infolge einer Ohroperation ohne Hirnerscheinungen und ohne Beteiligung der Sehnerven handelt. In allen anderen Fällen war jedoch der Sehnerv beteiligt, und zwar dürfen wir mit $\mathrm{v}$. Hippel annehmen, daB Stauungs. papillen oder ihre Folgezustände vorlagen. Leider ist aus den kurzen Krankengeschichten der aus der Literatur zusammengestellten Fälle nicht deutlich zu ersehen, inwieweit durch den LiquorabfluB Stauungspapille und Sehvermögen, wo es überhaupt noch vorhanden war, gebessert wurden. Die Allgemeinsymptome schwanden oder besserten sich in mehreren Fällen mit dem Auftreten des Nasenflusses erheblich und verschlimmerten sich wieder, sobald der AusfluB aufhörte. Dauerheilung seheint aber in keinem. Falle beobachtet $z u$ sein. Im Falle Caske ${ }^{12}$ ) kehrte das verlorene Gehör wieder.

Bei dem Patienten Feldhaus war der bisherige Ausgang ein selten glücklicher, indem nicht nur die schweren Allgemeinsymptome schwanden, sondern auch das Sehvermögen wiederkehrte. Es stieg rechts von Erkennen von Fingern in knapp $2 \mathrm{~m}$ a uf annähernd normalen Visus! Das Allgemeinbefinden besserte sich derart, daß Patient sich seit Jahreafrist wieder völlig gesund fühlt. Der Prolaps bildete sich erheblich zurück.

Der so selten glückliche Verlauf in unserem Falle wäre aber gar nicht möglich gewesen, wenn nicht frübzeitig trepaniert wäre, and darin liegt die weitere Bedeutung der Beobachtung. Wäre die Trepanation nicht gemacht, so wäre der Patient inzwischen sehr wahrscheinlich ad exitum gekommen, sicher aber völlig exblindet. Unsere Beobach. tungen bestaitigen also durchaus die Ansioht v. Hippels: ,DaB vor allen Dingen die Fälle, deren Grundleiden heilbar war, was sich meistens nicht voraussagen läßt, durch die Operation für die Dauer vollkommen gesund werden können, während sie ohne dieselbe sterben oder zum mindesten erblinden wrürden."

Die in unserem Falle beobachtete temporale Farbenhemianopsie weist auf einen Proze 3 in der Chiasmagegend hin. Der Durchbruch erfolgte vermutlich nach der Kellbeinhöhle, eine Annahme, die duroh die vorliegenden Sektionsbefunde gestützt wird. So ergab die Sektion bei dem Patienten Gutsches ${ }^{13}$ ) einen Tumor der Hypophyse, der den Knochen zerstörte. Es wurden an der Grenze zwischen Keil- and Siebbein zwei Öffnungen gefunden. Im ersten Falle Wollenbergs ${ }^{14}$ ) [Leber $\left.{ }^{15}\right)$ ] entwickelte sich infolge des Hydrocephalus ein Defekt in 
der Schädelbasis (Keil- oder Siebbein), im zweiten Fall bewirkten Tumoren im Hinterhauptslappen vermehrten Hirndruck, und es entstanden Dura- und Knochendefekte, die von der Schädelhöhle in die Nebenhöhlen der Nase führten. Auch bestanden Perforationen an den Vorderhörnern der Seitenventrikel, die sich nach den Duraund Knochendefekten öffneten. Auch Starck ${ }^{10}$ ) berichtet über Zerstörung der Dura und des Knochens an der Schädelbasis durch einen Tumor. Eine eigenartige Erklärung gibt Nothnagel7). Nach ihm gelangte die Flüssigkeit entlang den Nervenscheiden der Olfactorii in die Nase. Aus dem Sektionsbericht Baxters ${ }^{16}$ ) ist nicht ersichtlich, welohen Weg die Flüssigkeit nahm; die Hypophyse wird gar nicht erwähnt. Auch der Sektionsbefund Caskeysi1) gibt keinen klaren Aufschluß. Außer den erwähnten Ursachen wurde als Grundleiden gefunden: im Falle Starcks ${ }^{10}$ ) ein Tumor des Kleinhirnbrückenwinkels und im Falle Nothnagelsis) ein solcher der Vierhügel.

\section{Literaturverzeichnis.}

1) Hippel, E. v., Weitere Erfahrungen über die Ergebnisse der druckentlastenden Operationen bei der Stauungspapille. Arch. f. Augenheilk. 101, H. 4. 1920.- 2) Hi p pel, E. v., Die Krankheiten der Sehnerven. Handbuch der gesamten Augenheilkunde. 2. Aufl. 374. bis 397. Lieferung. II. Teil, X. Kap. B, Bd. $\%$ 1921. - ${ }^{3}$ ) Wolf, Fälle von Atrophie der Sehnerven mit spontanem AbfluB von Cerebrospinalflüssigkeit aus der Nase. Inaug.-Diss. Rostock 1907. - $\left.{ }^{4}\right)$ Elliotson, Med.'Times and Gaz. 1857. - 5) Paget, Transact. od the clin. Soe. 1857. - 9 Babinski, Stase papillaire guérie par la trépanation cran. Rev. d'ophtalmol. 1901, S. 692. - 7) Noth nagel, Geschwulst der Vierhügel, Hydrocephalus, Abtropfen von Cerebrospinalflïssigkeit durch die Nase. Wien. med. Blätter Nr. 6-8. 1888, - s) Emrys-Jones, Atrophy of the optic nerves associated with dropping of fluid from the nostril. Ophthalmic rev. 1888, 8. 95. - ${ }^{9}$ ) Lucae, 5 Wochon anhaltender profuser Ausfluß von Liquor cerebrospinalis ohne Hirnerscheinungen. Berl. klin. Wochenschr. 1899, Nr. 40. - $\left.{ }^{10}\right)$ Starok, Zur Pathologie der Gehirngeschwülste. Wanderversamml. d. südw. Neurologen. Juni 1907. - 11) Coppez, Deux cas d'amaurose arec hydrorrhée nasale. Arch. d'ophtalmol. 30, p. 334.1908.12) McCaskey, Report of case of tumour of the cerebellum with drainage of fluid through the nose. New York med. journ. r1, Nr. 13. 1900. - 13) Gutsche, Zur Pathogenese der Hypophysistumoren und über den nasalen Abfluß sowie das Verhalten des Liquor cerebrospinalis bei einer Struma pituitaria. Inaug.-Diss. Erlangen 1904. - 14) Wollenberg, Ein Fall von Hirntumor mit Abfluß von Cerebrospinalflüssigkeit durch die Nase. Arch. f. Psych. 31, H. 1 u. 2. 1898. $\left.{ }^{15}\right)$ Leber, Ein Fall von Hydrocephalus mit neurotischer Sehnervenatrophie und kontinuierlichem Abträufeln wässeriger Fliussigkeit aus der Nase. v. Graefes Arch. f. Ophthalmol. 29, I. Teil, S, 273. - ${ }^{16}$ ) Baxter, A case of paroxysmal clonic spasm of left rectus abdominis with symptoms pointing to the existence of gross intracranial disease. Brain 4, 525. 1882. 